\title{
Adaptação cultural da ferramenta de avaliação de comunicação em saúde (HCAT) para a língua portuguesa, Brasil
}

\author{
Cultural adaptation of the health communication assessment tool to \\ portuguese language, Brazil
}

\section{Adaptación cultural de la herramienta de evaluación de comunicación en salud (HCAT) para la lengua portuguesa, Brasil}

\author{
Nélida Beatriz Caldas dos Reis, a \\ be atriz.c@hotmail.com | https://orcid.org/0000-0001-7517-1478 \\ Fernanda Santos Nogueira Góes ${ }^{1, b}$ \\ fersngoes@eerp.usp.br | https://orcid.org/oooo-0001-6658-916X
}

Natália Del'Angelo Aredes ${ }^{2, c}$

nataliadel.aredes@gmail.com | https://orcid.org/0000-0002-1661-8601

Suzanne Hetzel Campbell ${ }^{3, d}$

suzanne.campbell@ubc.ca | https://orcid.org/o000-0002-8134-0669

\author{
${ }^{1}$ Universidade de São Paulo, Escola de Enfermagem de Ribeirão Preto. São Paulo, SP, Brasil. \\ ¿Universidade Federal de Goiás, Faculdade de Enfermagem. Goiânia, GO, Brasil. \\ 3Universidade da Colúmbia Britânica, Escola de Enfermagem. Vancouver, Canadá. \\ aMestrado em Enfermagem pela Universidade de São Paulo.

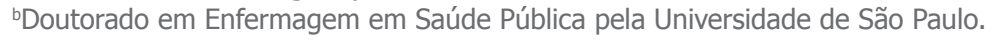 \\ 'Doutorado em Ciências pela Universidade de São Paulo. \\ dDoutorado em Enfermagem pela Universidade de Rhode Island.
}

\section{Resumo}

Este estudo tem como objetivo descrever o processo de adaptação cultural da ferramenta Health Communication Assessment Tool (HCAT) para o Brasil. A HCAT é uma escala que avalia comportamentos de comunicação em simulação clínica. Trata-se de uma pesquisa metodológica, desenvolvida em instituição de ensino superior por meio das seguintes etapas: tradução da ferramenta para a língua portuguesa; avaliação por um comitê de juízes; retrotradução; e avaliação semântica. Todas as etapas do processo de adaptação cultural foram rigorosamente seguidas, garantindo equivalências semânticas, idiomáticas, culturais e conceituais entre a versão original e a brasileira, além de concordância superior a $70 \%$ dos professores de enfermagem das regiões Nordeste, Sudeste e Sul que participaram da última etapa, ou seja, da avaliação semântica. Conclui-se que a versão brasileira do HCAT foi adaptada culturalmente e poderá avaliar a habilidade de comunicação de estudantes de enfermagem em cenário de simulação clínica.

Palavras-chave: Educação em enfermagem; Comunicação em Saúde; Segurança do paciente; Treinamento por simulação; Estudos de validação. 


\begin{abstract}
This study aims to describe the process of cultural adaptation of the Health Communication Assessment Tool (HCAT) to Brazil. The HCAT is a scale that evaluates communication behaviors in clinical simulation. We developed a methodological research in an institution of higher education through the following stages: translation of the tool from English into Portuguese; evaluation by a committee of judges; back-translation; and semantic evaluation. All stages of the cultural adaptation process were rigorously developed ensuring semantic, idiomatic, cultural and conceptual equivalence between the original and the Brazilian version, in addition to a concordance of more than $70 \%$ of the nursing teachers who were invited to collaborate in evaluation. They are from three Brazilian regions - Northeast, Southeast and South - and participated in the last stage, namely semantic evaluation. It was concluded that the Brazilian version of the HCAT was culturally adapted and it will be a good tool to assess the communication skills of nursing students in a scenario of clinical simulation.
\end{abstract}

Keywords: Nursing education; Health Communication; Patient safety; Training by simulation; Validation studies.

\title{
Resumen
}

Este estudio se centra en describir el proceso de la adaptación cultural de la herramienta Health Comunnication Assessment Tool (HCAT) a Brasil. El HCAT es una escala que evalúa la comunicación de los comportamientos en simulación clínica. El estudio constituye una investigación metodológica desarrollada en una institución de educación superior a través de las siguientes etapas: la traducción de la herramienta al portugués; evaluación por un comité de jueces; back-translation; y evaluación semántica. Todas las etapas del proceso de adaptación cultural fueron desarrolladas con el debido rigor garantizando equivalencias semánticas, idiomáticas, culturales y conceptuales entre la versión original y la brasileña, además de concordancia superior al 70\% de los profesores de enfermería de las regiones Nordeste, Sudeste y Sur del Brasil que participaron de la última etapa, es decir, la evaluación semántica. Se consideró que la versión brasileña de la HCAT fue adaptada culturalmente y podrá evaluar las habilidades de comunicación de los estudiantes de enfermería en escenario de simulación clínica.

Palabras clave: Educación en enfermería; Comunicación en Salud; Seguridad del paciente; Entrenamiento a través de simulación; Estudios de validación.

Contribuição dos autores:

Concepção e desenho do estudo: Nélida Beatriz Caldas dos Reis e Fernanda Santos Nogueira Góes.

Aquisição, análise ou interpretação dos dados: Nélida Beatriz Caldas dos Reis e Fernanda Santos Nogueira Góes.

Redação do manuscrito: Nélida Beatriz Caldas dos Reis, e Fernanda Santos Nogueira Góes.

Revisão crítica do conteúdo intelectual: Natália Del'Angelo Aredes e Suzanne Hetzel Campbell.

Declaração de conflito de interesses: Este trabalho não apresenta conflito de interesses.

Fontes de financiamento: Não há.

Considerações éticas: A pesquisa foi autorizada pelo Comitê de Ética envolvendo Seres Humanos, CAAE 41472415.2.0000.5393 e seguiu as recomendações éticas nacionais e internacionais.

Agradecimentos/Contribuições adicionais: Não há.

Histórico do artigo: Submetido: 28.fev.2018 | Aceito: 21.out.2018 | Publicado: 21.dez.2018.

Apresentação anterior: Não houve.

Licença CC BY-NC atribuição não comercial. Com essa licença é permitido acessar, baixar (download), copiar, imprimir, compartilhar, reutilizar e distribuir os artigos, desde que para uso não comercial e com a citação da fonte, conferindo os devidos créditos de autoria e menção à Reciis. Nesses casos, nenhuma permissão é necessária por parte dos autores ou dos editores. 


\section{Introdução}

A comunicação é parte integrante da educação em enfermagem, pois é um componente fundamental das atividades dessa área e relações humanas ${ }^{1,2}$, tendo em vista que, por meio dela, paciente e família podem emitir informações valiosas para a condução terapêutica pela equipe de saúde ${ }^{3,4}$. Trata-se de uma competência que tem recebido notável atenção na última década ${ }^{5}$.

Diversos documentos internacionais ${ }^{6-10}$ e nacionais ${ }^{11,12}$ têm advogado a favor da qualidade e da efetividade da comunicação do aluno com os pacientes como um dos aspectos fundamentais na formação de enfermeiros. Nesse sentido, os enfermeiros devem ser capazes de lidar com pacientes e suas famílias com sensibilidade, empatia e responsabilidade moral, além de estarem aptos a ensiná-los e a orientá-los sobre os aspectos da terapia em si, das ações de prevenção e da própria navegação no sistema de saúde ${ }^{8}$.

Para tanto, diversos países ${ }^{6-12}$ consideram que a comunicação deve ser reconhecida como uma competência essencial para os líderes no campo e como uma das habilidades transversais para o desenvolvimento profissional do enfermeiro na prática efetiva de sua profissão. Nesse contexto, ensiná-la requer mudanças significativas no currículo, nas metodologias de aprendizagem e na forma de se fazer avaliação ${ }^{8,11,12}$.

A comunicação pode ser definida como o compartilhamento de informações por meio verbal, não verbal e por escrito, por meio da qual os comunicadores eficazes têm a capacidade de transmitir informações (o que está sendo dito) aos outros e, ao mesmo tempo, recebê-las de outras pessoas de maneira eficaz ${ }^{13}$. Ademais, a comunicação deve reconhecer a importância de cada ser humano para que ele seja tratado como um sujeito com integridade, liberdade e direito à autonomia ${ }^{3,4}$.

Embora a enfermagem esteja bem posicionada na linha assistencial para defender os pacientes e prevenir erros de comunicação $0^{14}$, a falta de assertividade e hesitação em falar pode ser um problema recorrente para a segurança do paciente ${ }^{15,16}$. Nos Estados Unidos, erros de comunicação foram identificados como causa de 1.796 eventos sentinela entre 2013 e $2015^{17}$.

Nessa perspectiva, a comunicação eficaz entre os profissionais de saúde torna-se criticamente importante para a segurança do paciente e para a qualidade do cuidado ${ }^{18-20}$, sendo determinante para o nível de satisfação dos pacientes ${ }^{21,22}$. Eficácia que influencia diretamente na adesão e recuperação dos mesmos, reduzindo a angústia e a ansiedade psicológica ${ }^{19}$ de forma a possibilitar ainda a garantia de manutenção da dignidade desses pacientes ${ }^{19}$.

Contudo, as habilidades eficazes de comunicação continuam sendo uma barreira para os enfermeiros recém-formados que trabalham no sistema de saúde ${ }^{23,24}$, provavelmente por causa de suas percepções dada a sua inexperiência profissional e por acreditarem ter limitações do conhecimento requerido para a prática ${ }^{15}$.

Muitas vezes, os enfermeiros não possuem as habilidades de comunicação necessárias para fazê-la de maneira eficaz, possivelmente por não terem recebido educação nesse tema durante a formação inicial em seu curso de graduação ${ }^{13,25}$. Estudos têm evidenciado que os estudantes de enfermagem perceberam a importância da comunicação individualizada e a necessidade de serem ativos em sua aprendizagem ${ }^{26}$, muito embora tenham sentido falta de habilidades relevantes para estabelecer comunicação eficaz com os pacientes e seus familiares ${ }^{13,26}$.

Diante do exposto, tudo indica que a eficácia dessa competência é a base para o cuidado de enfermagem seguro $^{20}$. No entanto, o seu desenvolvimento não é algo que possa ser considerado intuitivo; de maneira que deve ser considerado conteúdo de ensino ${ }^{27}$.

Acresce-se a isso que o ambiente de aprendizagem, a complexidade dos pacientes e os recursos organizacionais são contextos importantes para facilitar ou dificultar o desenvolvimento da habilidade de comunicação pelos futuros enfermeiros ${ }^{28}$, ou seja, o exercício constante dessa competência, com apoio de um facilitador, e a utilização de recursos de ensino adequados podem estimular a aprendizagem do aluno em relação ao tema. 
No que tange a esse cenário, a educação em todo o mundo está passando por mudanças aceleradas e os métodos de ensino estão evoluindo ${ }^{29}$. Dessa forma, descobrir as melhores práticas de ensinar e aprender essas habilidades no curso de graduação em enfermagem é fundamental para auxiliar a transição do aluno para a vida profissional ${ }^{30}$.

Nesse sentido, constata-se a necessidade de mudança nas abordagens pedagógicas utilizadas para garantir desenvolvimento de conhecimento significativo para os estudantes de enfermagem ${ }^{23,31}$, posto que a comunicação ideal ocorre em um relacionamento dialógico, em que a transmissão e a verticalização na interação entre as pessoas são elementos indesejáveis, sendo preciso, portanto, a transição da perspectiva tradicional para uma abordagem mais inclusiva e horizontal ${ }^{3,4}$.

Oportunidades para que os alunos possam processar, praticar e aperfeiçoar a comunicação centradas no paciente, usando linguagem comum, é um componente-chave do currículo de enfermagem ${ }^{23,30}$, considerando também modelos ativos de ensino e aprendizagem ${ }^{32}$.

Entre os métodos ativos de ensino, a simulação clínica pode ser utilizada pelo futuro enfermeiro como um recurso de aprendizagem e desenvolvimento de habilidades não técnicas em um ambiente controlado e seguro que reproduza situações da vida real5,8, e tem sido associada a um aumento na percepção da autoeficácia nas comunicações ${ }^{19}$.

A simulação clínica é definida como atividade ou evento que replica o ambiente da prática clínica ${ }^{33} \mathrm{e}$ integra o mundo real ao processo de ensino-aprendizagem, de modo a atingir os objetivos de ensino ${ }^{34}$.

Diante da importância da comunicação para a formação do enfermeiro e para o cuidado destinado ao paciente e à sua família, a possibilidade do desenvolvimento e/ou treinamento da habilidade de comunicação em ambientes simulados, deparou-se com a HCAT - Health Communication Assessment Tool (ferramenta de avaliação da comunicação em saúde) ${ }^{35}$.

A HCAT foi desenvolvida por pesquisadores americanos a partir da análise de gravações de simulações de 350 estudantes de graduação em enfermagem ${ }^{35}$. A versão atualizada da HCAT contém 22 afirmações analisadas a partir de uma escala que varia de um a cinco pontos, sendo: 1 - concordo fortemente; 2 - concordo; 3 - indeciso; 4 - discordo; 5 - discordo fortemente. A ferramenta identifica se os comportamentos (verbal ou não verbal) foram realizados como evidência de comunicação e aspectos como estabelecimento de vínculo, valorização/estímulo à autonomia do paciente, educação/capacitação do paciente, empatia e evitando falhas de comunicação. É composta ainda por uma questão aberta para que o facilitador possa identificar o uso de terminologia de saúde ou jargões.

Os recursos de ensino podem auxiliar no desenvolvimento da competência dos alunos na comunicação, desde que o professor facilitador tenha oportunidades de oferecer feedback ao estudante para que ele participe ativamente do processo ${ }^{36}$. Tendo em vista a pouca divulgação de estudos brasileiros que subsidiem o ensino de comunicação em ambientes simulados atrelados à necessidade de instrumentos para avaliar a comunicação de estudantes de enfermagem disponíveis em língua portuguesa, o presente estudo propõese a descrever o processo de adaptação cultural da ferramenta Health Communication Assessment Tool (HCAT) para o Brasil.

\section{Método}

Trata-se de um estudo metodológico ${ }^{37}$, para adaptação cultural, da ferramenta HCAT a partir de sua versão anterior em língua inglesa americana, desenvolvido em universidade pública do estado de São Paulo, Brasil, entre junho de 2015 e janeiro de 2017.

Para a adaptação cultural foi adotado o processo metodológico ${ }^{38}$ composto pelas seguintes fases: tradução inicial para língua portuguesa (consenso: Versão I, em português brasileiro); avaliação por um comitê de juízes (Versão II, em português brasileiro); retrotradução (back-translation); avaliação semântica dos itens 
(Versão III, em português brasileiro). Futuramente, deverão ser realizados o pré-teste (Versão final, em português brasileiro) e a análise da medida adaptada por meio de validação e testes de confiabilidade, para garantir a precisão e estabilidade da escala ${ }^{i}$.

De acordo com o referencial metodológico, fez-se necessária a participação de docentes de enfermagem em duas etapas distintas:

- Comitê de juízes - avaliação da tradução e retrotradução: foram convidadas cinco docentes de enfermagem que atenderam aos critérios de inclusão: experiência de, no mínimo, três anos na docência em enfermagem; fluência na língua inglesa; atividades de ensino, pesquisa ou extensão sobre o tema comunicação e/ou adaptação cultural de instrumentos/ferramentas/escalas;

- Avaliação semântica: foram convidados 49 docentes de enfermagem das cinco regiões brasileiras na tentativa de que o instrumento fosse analisado por profissionais com diferentes experiências; esses profissionais foram identificados na Plataforma Lattes do Conselho Nacional de Desenvolvimento Científico e Tecnológico (CNPq). Os critérios de inclusão foram: experiência clínica, ensino e/ou capacitação em comunicação e ensino e/ou capacitação em simulação.

\section{Processo de adaptação cultural da ferramenta HCAT}

\section{Tradução inicial para a língua portuguesa (Brasil)}

A etapa de tradução do HCAT para a língua portuguesa foi realizada por dois tradutores de forma independente. As duas versões traduzidas foram comparadas, selecionando-se as frases de melhor expressão de cada uma delas, dando origem à primeira versão consensual em língua portuguesa PVCP-1.

\section{Avaliação por comitê de juízes}

Na segunda etapa, a ferramenta traduzida PVCP-1 foi avaliada por um comitê de juízes em busca de uma segunda versão consensual brasileira. Foram consideradas as equivalências semântica (significado das palavras), idiomática (substituição de expressões e coloquialismos por expressões equivalentes na cultura local), conceitual (analisa se um dado domínio do instrumento tem o mesmo valor, tanto na cultura da versão original do instrumento, quanto na cultura para a qual está sendo adaptado) e cultural (adequação das expressões do instrumento original às do idioma para o qual o instrumento será retrotraduzido). A reunião do comitê de juízes foi realizada por duas horas. Cada participante recebeu a versão original em idioma inglês e a primeira versão PVCP-1. Todos os itens tiveram mais de $80 \%$ de concordância entre os juízes, dando origem assim à segunda versão consensual em língua portuguesa SVCP-2.

\section{Retrotradução}

A retrotradução da SVCP-2 foi solicitada a dois tradutores independentes uma versão para o inglês americano. Após a análise das versões, gerou-se a primeira versão consensual retrotraduzida PVCR-1 do HCAT, que foi encaminhada para os autores; estes excluíram duas afirmações, sob a justificativa de que o HCAT tinha sido recentemente revisto. A partir de então foi utilizada a terceira versão consensual em língua portuguesa TVCP-3 do HCAT, avaliada na ocasião pelos autores originais.

\footnotetext{
i Após este estudo, HCAT é considerada adaptada ao contexto brasileiro e deverá ser submetida a etapas posteriores de análise de validade e confiabilidade, preferencialmente com múltiplos momentos de testes e diferentes amostras.
} 


\section{Avaliação semântica}

Na etapa de avaliação semântica, foi solicitado aos docentes participantes que responderam ao e-mail aceitando o convite, que avaliassem o instrumento e, se necessário, fizessem sugestões de mudanças na redação para melhor entendimento do público-alvo. Para realizar a avaliação semântica (equivalência de significado das palavras), cada afirmação do TVCP-3 do HCAT foi analisada de acordo com o grau de concordância, por meio da escala: 1 - discordo fortemente; 2 - discordo; 3 - concordo; e 4 - concordo fortemente.

A análise das propriedades da medida adaptada será realizada em estudo posterior.

\section{Resultados}

\section{Tradução inicial para a língua portuguesa}

De posse das duas traduções da HCAT, as pesquisadoras compararam as duas versões na busca das melhores afirmações de cada uma delas. Assim, foi construída a primeira versão da ferramenta HCAT PVCP, composta por 16 afirmações da Versão 1 e cinco da Versão 2, sendo que as afirmações 4, 9, 11 e 20 foram idênticas.

\section{Avaliação pelo comitê de juízes}

Na etapa de avaliação pelo comitê de juízes, participaram cinco docentes de enfermagem. Todas têm de três a 14 anos de atuação na docência, são fluentes na língua inglesa e ministram atividades teóricas e práticas sobre comunicação. Entre elas, quatro realizaram pesquisas sobre comunicação ou adaptação cultural de instrumentos para o Brasil e realizaram atividades de extensão sobre comunicação. Na reunião presencial, cada item da versão original e da PVCP-1 foi discutido pontualmente, sendo na oportunidade verificadas as equivalências entre a versão original e a brasileira.

Além de alterações no título da HCAT, foram também realizadas substituições de termos apropriados para o Brasil.

\section{Retrotradução}

Na etapa de retrotradução, a versão SVCP-2 foi encaminhada aos tradutores para que realizassem a tradução para o idioma inglês americano.

A partir do que foi disponibilizado pelos tradutores, chegou-se à retrotradução da HCAT, posteriormente enviada aos autores originais a fim de garantir o rigor metodológico e a preservação do conteúdo inicial da versão original.

Os autores concordaram com a versão retrotraduzida, contudo sugeriram a redução do número de afirmações da HCAT de 24 para 22, de forma que foram então suprimidas as afirmações 20 e 25. 
Assim, obedecendo-se ao rigor metodológico, procederam-se os seguintes passos novamente:

- Versão reduzida (considerando também os ajustes após os questionamentos feitos pelo comitê de juízes) traduzida para o português brasileiro; cabe observar que não houve diferenças entre as versões;

- Geração de nova retrotradução;

- Envio da versão retrotraduzida aos autores da versão original americana: aprovada.

Ao final dessa etapa, foi gerada a terceira versão consensual em língua portuguesa: SVCP-3.

\section{Avaliação semântica}

Na etapa de avaliação semântica dos itens da SVCP-3 da HCAT, participaram dez docentes de enfermagem do Brasil. Cabe destacar que a solicitação foi enviada duas vezes, em um período de 90 dias, para 49 docentes.

Entre as dez participantes, nove eram enfermeiras e uma pedagoga que, porém, atuava no ensino de enfermagem. Tinham de cinco a 26 anos de atuação na docência, todas com experiência de ensino teórico e prático sobre comunicação, sendo que uma delas ensina conteúdo específico sobre comunicação. Todas pesquisavam comunicação ou adaptação cultural de instrumentos e duas orientavam atividades de extensão sobre comunicação em saúde.

Os resultados das avaliações estão descritos na Tabela 1. Verificou-se que cada um dos itens da versão brasileira do HCAT obteve concordância mínima superior a 80\%, com exceção do item 8 que obteve 70\% de concordância. 
Tabela 1 - Resultados da avaliação semântica acerca da versão final brasileira da HCAT

\begin{tabular}{|c|c|c|c|c|c|}
\hline Afirmações (HCAT) & $\begin{array}{l}\text { Discordo } \\
\text { fortemente } \\
(\%)\end{array}$ & $\begin{array}{l}\text { Discordo } \\
(\%)\end{array}$ & $\begin{array}{l}\text { Indeciso } \\
(\%)\end{array}$ & $\begin{array}{l}\text { Concordo } \\
(\%)\end{array}$ & $\begin{array}{l}\text { Concordo } \\
\text { fortemente } \\
(\%)\end{array}$ \\
\hline 1. O estudante/profissional apresentou-se ao paciente e/ou família. & & & & 30 & 70 \\
\hline 2. O estudante/profissional apertou a mão do paciente e/ou família ou cumprimentou apropriadamente. & & 20 & & 30 & 50 \\
\hline 3. O estudante/profissional explicou a razão de sua visita em termos apropriados. & & 10 & & 10 & 80 \\
\hline 4. O estudante/profissional usou comunicação positiva, incluindo um sorriso para encorajar as interações. & & & 10 & 20 & 70 \\
\hline 5. O estudante/profissional manteve contato visual enquanto conversava com o paciente e/ou família. & & & & 20 & 80 \\
\hline 6. O estudante/profissional comunicou o que estava prestes a fazer ANTES de fazê-lo. & & & 20 & & 80 \\
\hline $\begin{array}{l}\text { 7. O estudante/profissional perguntou ao paciente ou familiar se poderia tocar o paciente ANTES de fazer um } \\
\text { procedimento ou exame (pressão arterial, ausculta, punção venosa, sondagem etc). }\end{array}$ & & & 10 & 10 & 80 \\
\hline 8. O estudante/profissional tocou o paciente apropriadamente. & & 10 & 20 & 20 & 50 \\
\hline 9. O estudante/profissional passou a maior parte do tempo perto do paciente. & & & 20 & 10 & 70 \\
\hline 10. O estudante/profissional sentou-se ao orientar ou conversar com o paciente. & & & 20 & 10 & 70 \\
\hline 11. O estudante/profissional ouviu mais do que falou. & & & & 20 & 80 \\
\hline 12. O estudante/profissional inclinou-se em direção à pessoa que falava para demonstrar interesse. & & & 10 & 10 & 80 \\
\hline 13. O estudante/profissional orientou efetivamente o paciente e/ou família sobre o procedimento, doença e/ou tratamento. & & & & 40 & 60 \\
\hline 14. O estudante/profissional fez perguntas para encorajar o feedback e aumentar a clareza. & 10 & & & 20 & 70 \\
\hline $\begin{array}{l}\text { 15. O estudante/profissional reconheceu e respondeu apropriadamente aos comportamentos verbais e não verbais } \\
\text { (franzir de testa, lágrimas, histeria, silêncio etc.) do paciente e/ou família. }\end{array}$ & & & 20 & 30 & 50 \\
\hline 16. O estudante/profissional usou tom de voz e volume apropriados para a situação. & & 10 & 10 & & 80 \\
\hline $\begin{array}{l}\text { 17. O estudante/profissional evitou julgar comportamentos do paciente/família (ex: condição econômica, abuso, } \\
\text { uso de drogas, orientação sexual, religião/diferenças culturais etc.). }\end{array}$ & & & 10 & 10 & 80 \\
\hline $\begin{array}{l}\text { 18. O estudante/profissional passou um tempo igual ou maior abordando aspectos psicossociais no cuidado ao } \\
\text { paciente/família em relação aos aspectos clínicos (biológicos). }\end{array}$ & & & & 20 & 80 \\
\hline $\begin{array}{l}\text { 19. O estudante/profissional perguntou sobre os sentimentos do paciente/família sobre a situação, demonstrando } \\
\text { preocupação. }\end{array}$ & & & 20 & 10 & 70 \\
\hline $\begin{array}{l}\text { 20. O estudante/profissional reconheceu o conflito e tentou obter informações e encontrar oportunidades para } \\
\text { minimizá-lo ou manejá-lo. }\end{array}$ & & & 20 & 20 & 60 \\
\hline $\begin{array}{l}\text { 21. O estudante/profissional desenvolveu, manteve ou aprimorou relacionamento interpessoal com o paciente e/ } \\
\text { ou família (via comunicação e profissionalismo). }\end{array}$ & & & 10 & 50 & 40 \\
\hline $\begin{array}{l}\text { 22. O estudante/profissional evitou termos técnicos de saúde (sinais vitais, punção venosa etc.) [inclua abaixo os } \\
\text { termos técnicos utilizados]. }\end{array}$ & & & 20 & 10 & 70 \\
\hline Se termos técnicos foram utilizados, liste-os: & & & & & \\
\hline
\end{tabular}

Fonte: Os autores (2018). 


\section{Discussão}

Neste estudo, realizamos a adaptação cultural da ferramenta HCAT, para o idioma português brasileiro.

Nos últimos anos, tem aumentado a demanda pela adoção de instrumentos/ferramentas que garantam avaliação clara e comunicável de uma situação, bem como que colaborem para a qualidade e segurança da assistência ao paciente e para a diminuição de custos de tratamento ${ }^{35,37}$.

Nesse sentido, a adoção sistemática de instrumentos, ferramentas ou escalas para avaliação das necessidades dos pacientes e da equipe de saúde pode facilitar o processo decisório dos profissionais de saúde ${ }^{35,37}$.

O processo adotado para tradução para a língua portuguesa e a adaptação cultural da HCAT seguiram o rigor científico, utilizando-se de etapas empregadas em vários outros estudos realizados no Brasil ${ }^{39-41}$, assim como o presente estudo que buscou realizar a adaptação cutural e validade de conteúdo. Entretanto, pretende-se que estudos adicionais sejam conduzidos de forma a manter a continuidade das avaliações das propriedades psicométricas.

O processo de adaptação cultural tem sido uma atividade reconhecida como importante no intuito de proporcionar informações sobre saúde ou educação que possam ser generalizadas, respeitando-se as características de cada país. Permite a elaboração de instrumento conceitualmente equivalente ao original e culturalmente aceitável pelo país para o qual foi adaptado ${ }^{39-42}$. Assim, no presente estudo buscou-se obter medidas que sejam igualmente apropriadas e validadas para as culturas americana e brasileira.

Apesar de não haver consenso universal sobre a forma de adaptar culturalmente uma ferramenta para uso em outra cultura, há consenso sobre a inadequação de apenas traduzi-lo sem que seja realizada sua adaptação cultural ${ }^{42}$. Ademais, não ajustar sua terminologia à população-alvo, incluir ou excluir itens $\mathrm{e}$ não submeter a ferramenta adaptada à análise pode comprometer, de forma importante, a qualidade da informação e a capacidade de generalização dos resultados ${ }^{37}$.

$\mathrm{Na}$ etapa de tradução inicial da HCAT para a língua portuguesa, optou-se intencionalmente que os tradutores fossem naturais do país que receberia a tradução de forma que detivessem o domínio semântico, conceitual e cultural do idioma da ferramenta original. Nessa perspectiva, torna-se imprescindível que um dos tradutores tenha noção sobre os objetivos básicos da ferramenta, pois, assim, as peculiaridades do tema estudado serão respeitadas e a perspectiva clínica será mais adequada. Sua tradução oferecerá linguagem usada pela população em geral, esclarecendo as ambivalências que os termos do idioma original da ferramenta podem apresentar. Outra recomendação é de que os tradutores utilizem uma linguagem que possa ser compreendida pela população em geral ${ }^{42,43}$.

Para avaliação pelo comitê de juízes, salienta-se que a existência de um grupo presencial para discutir as traduções permitiu o debate exaustivo de cada item visando ao consenso do grupo. A discussão em grupos permite a interação entre as pessoas na fase de obtenção de dados necessários à pesquisa, a partir de ambiente favorável, propiciando espaços para que os participantes manifestem suas percepções e pontos de vista $^{3}$ e abreviem o tempo a ser dispensado nesse tipo de pesquisa ${ }^{37}$.

Devido às dúvidas que surgiram durante a etapa do comitê de juízes, alguns questionamentos foram enviados aos autores da versão americana da HCAT. Entre as dúvidas enviadas pelos pesquisadores e as modificações propostas pelos autores, é oportuno mencionar a exclusão da expressão 'tocou o paciente de maneira apropriada' e o acréscimo da expressão 'ou cumprimentou apropriadamente'. Outrossim, foi discutida com os autores da versão original a mudança do termo 'educar' por 'orientar', o que foi aceito.

Finalmente, o comitê de juízes sugeriu que deveria haver clareza sobre quais tipos de comportamentos poderiam ser considerados como comportamentos estereotipados. Após discussão com os autores, novas modificações foram realizadas. Em vez de 'evitou estereotipar comportamentos' (como, por exemplo: mãe adolescente, mãe solteira, etnia, orientação sexual, gênero, ou diversidades econômicas, entre outros), 
optou-se pelo uso de 'o estudante/profissional' evitou julgar comportamentos do paciente/família (ex.: condição econômica, abuso, uso de drogas, orientação sexual, religião/diferenças culturais, entre outros)'.

Na etapa da avaliação semântica dos itens da terceira versão consensual em português SVCP-3 da HCAT, poucos docentes propuseram-se a colaborar. Supostamente, uma das justificativas pode estar atrelada ao excesso de atividades de ensino, pesquisa, extensão e gestão exercidas pelos docentes universitários. O produtivismo acadêmico tem sido importante fator de intensificação da sobrecarga de trabalho ao docentepesquisador, cada vez mais incumbido de intensas atividades e, muitas vezes, isento de autonomia ${ }^{44}$. Possivelmente, tal sobrecarga motivou a demora na aceitação e a recusa de vários dos docentes convidados a participarem da etapa em questão.

Das dez docentes participantes, quatro atuam na Região Sul, duas na Região Nordeste e quatro na Sudeste. Dada à extensão geográfica do Brasil, a qual confere ao país diferentes identidades culturais e sociais, a participação de docentes de distintas localidades do Brasil permitiu que houvesse maior credibilidade à versão brasileira do HCAT.

Pôde-se constatar, ainda, que apenas uma delas possui formação acerca da temática 'comunicação', denotando o quanto as habilidades de comunicação podem não estar sendo avaliadas e desenvolvidas sistematicamente, durante a formação dos graduandos e pós-graduandos ${ }^{6-12}$.

No que concerne às sugestões de algumas avaliadoras, cabe destacar a afirmação 'O estudante/ profissional tocou o paciente apropriadamente', a partir da qual foram emitidos os seguintes comentários:

'O que é tocar apropriadamente? Levar em conta cada paciente....; ‘Fiquei em dúvida se apropriadamente não poderia ser trocado por "respeitosamente" ou de "maneira respeitosa"....

No Brasil, o toque apropriado é confortador e faz parte ativa do cuidado ${ }^{3,46}$. Entretanto, cabe ao enfermeiro estabelecer interação com o paciente para quem o toque pode ser compreendido para além de uma atitude procedimental, ou seja, o enfermeiro deve buscar a comunicação empática sabendo do potencial afetivo do toque ${ }^{3,45}$.

Apesar das variações que podem existir entre diferentes países, na cultura ocidental os toques nos membros superiores, ombro, braço, antebraço e mãos são mais aceitáveis do que em áreas habitualmente cobertas por vestes e, consequentemente, mais protegidas, tais como, tórax, abdômen e pernas ${ }^{45}$.

De maneira geral, na cultura brasileira, durante um diálogo estabelecido entre profissional e paciente, toques nos braços são ‘tolerados' e não são vistos como desrespeitosos. Apesar de serem mais usuais os toques nos braços, em ambiente hospitalar pode haver uma 'permissão implícita', que permite tocar, por exemplo, o abdômen dos pacientes. Entretanto, cabe ao profissional de saúde solicitar autorização para exercer esse tipo de ação profissional ${ }^{35,45}$.

A sugestão de incluir o termo 'durante os procedimentos' na frase 'O estudante/profissional tocou o paciente apropriadamente' não foi acatada uma vez que se depreendeu que a modificação poderia denotar a ideia do toque associado apenas ao procedimento técnico, afastando-se da concepção do toque como uma forma de cuidado de enfermagem ${ }^{35,45}$.

A partir dos resultados deste estudo, espera-se colaborar para a formação em enfermagem e, uma vez que a comunicação permeia todas as ações do aluno e futuro enfermeiro, possibilite o desenvolvimento de suas habilidades relativas a ela durante o aprendizado, dada a sua importância futura para a prática.

\section{Conclusão}

O presente estudo realizou a adaptação cultural da ferramenta HCAT para o português brasileiro, perfazendo diferentes etapas de acordo com os rigores científico e metodológico. 
A versão brasileira da HCAT pode ser utilizada no contexto brasileiro, embora ainda se faça necessário realizar o pré-teste e a análise das medidas adaptadas utilizando uma amostra representativa de professores/ facilitadores de simulação clínica em enfermagem.

Dada a importância da comunicação para a formação do enfermeiro e, consequentemente, para o cuidado ao paciente, família e sociedade, espera-se que quando disponibilizada para uso, no ensino de enfermagem, a versão brasileira da HCAT possa ser uma estratégia para avaliar a comunicação de estudantes de enfermagem, bem como uma ferramenta de apoio ao professor em ambientes de simulação.

\section{Referências}

1. McGilton K, Irwin-Robinson H, Boscart V, Spanjevic L. Communication enhancement: nurse and patient satisfaction outcomes in a complex continuing care facility. J Adv Nurs [Internet]. 2006 [cited 2018 jun 28];54(1):35-44. doi: http://dx.doi.org/10.1111/j.1365-2648.2006.03787.x

2. O'Hagan S, Manias E, Elder C, Pill J, Woodward-Kron R, McNamara T, et al. What counts as effective communication in nursing? Evidence from nurse educators' and clinicians' feedback on nurse interactions with simulated patients. ] Adv Nurs [Internet]. 2014 [cited 2018 jun 28]; 70(6):1344-55. doi: https:// doi.org/10.1111/jan.12296

3. Stefanelli MC. A comunicação nos diferentes contextos da enfermagem. São Paulo: Pearson Education; 2010.

4. Tamaki CM, Meneguin S, Alencar RA, Luppi CHB. Cuidar de pacientes terminais: recepção dos enfermeiros de uma unidade de terapia intensiva de hospital público. Invest Educ Enferm. 2014; 32(3):414-20.

5. Defenbaugh $\mathrm{N}$, Chikotas NE. The outcome of interprofessional education: Integrating communication studies into a standardized patient experience for advanced practice nursing students. Nurse Education in Practice [Internet]. 2016 [cited 2018 jun 28];16:176e-81. doi: http://dx.doi.org/10.1016/j. nepr.2015.06.003.

6. American Association of Colleges of Nursing [Internet]. The essentials of baccalaureate education for professional nursing practice; 2008 [cited 2018 jun 28]. Available from: http://www.aacn.nche.edu/ education-resources/BaccEssentials08.pdf.

7. Research MoEa. National Curriculum for nursing education in Norway. Oslo: Rammeplan for sykepleierutdanning; 2008.

8. Bauchat JR, Seropian M, Jeffries PR. Communication and empathy in thepatient-centered care model - why simulation-based training is not optional. Clin Simul Nurs [Internet]. 2016 [cited 2018 jun 28];12(8):356-9. doi: http://dx.doi.org/10.1016/j.ecns.2016.04.003

9. Parliamentary and Health Service Ombudsman United Kingdom The Ombudsman's Annual Report and Accounts 2016-2017 [Internet]. London: Williams Lea Group; 2017 [cited 2018 jun 28]. Available from: https://www.gov.uk/government/publications.

10. Australian Commission on Safety and Quality in Health Care. Annual Report 2016-17 [Internet]. Sydney: ACSQHC; 2017 [cited 2018 jun 28]. Available from: https://www.safetyandquality.gov.au

11. Ministério da Saúde (BR). Conselho Nacional de Saúde. Resolução n 569, de 08 de dezembro de 2017: Princípios e diretrizes comuns para a graduação na área da saúde- CNS. DOU [Internet]. 2018 fev. 26 [citado em 28 jun. 2018];85-90. Disponível em: https://goo.gl/GYZp6R

12. Ministério da Educação (BR). Conselho Nacional de Educação. Câmara Superior de Educação. Resolução CNE/CES n 3, de 7/11/2001. Institui Diretrizes curriculares nacionais do curso de graduação em enfermagem. DOU [Internet]. 2001 nov. 09 [citado 28 jun. 2018]. Disponível em: http://portal.mec.gov. br/cne/arquivos/pdf/CES03.pdf.

13. Shlafer RJ, McRee A-L, Gower AL, Bearinger LH. Better Communication for Better Public Health: Perspectives From an Interdisciplinary Training Program. Health Promotion Practice [Internet]; 2016 [cited 2018 jun. 28];17(2):165-8. doi: http://dx.doi.org/10.1177/1524839915627453

14. Rainer J. Speaking up: factors and issues in nurses advocating for patients when patients are in jeopardy. J. Nurs. Care Qual [Internet]. 2015 [cited 2018 jun. 28];30:53-62. doi: https://doi. org/10.1097/NCQ.0000000000000081 
15. Omura A, Stoneb TE, Maguirec J, Levett-Jones T. Exploring Japanese nurses' perceptions of the relevance and use of assertive communication in healthcare: A qualitative study informed by the Theory of Planned Behaviour. Nurse Educ Today [Internet]. 2018 [cited 2018 nov 12];67:100-7. doi: https://doi. org/10.1016/j.nedt.2018.05.004

16. Eggertson L. On the same team? Nurse-physician communication. Can. Nurse [Internet]. 2012 [cited 2018 jun 28];108(5):28-32.

17. The Joint Commission. Sentinel Event Statistics Data: Event Type by Year (1995-Q2-2016) [Internet]. 2017 [cited 2018 jun 28]. Available from: https://www.jointcommission.org/se data event type by year $/$

18. Gausvik C, Lautar A, Miller L, Pallerla H, Schlaudecker J. Structured nursing communication on interdisciplinary acute care teams improves perceptions of safety, efficiency, understanding of care plan and teamwork as well as job satisfaction. J Multidiscip Healthc [Internet]. 2015 [cited 2018 jun. 28];8:33-7. doi: https://doi.org/10.2147/JMDH.S72623

19. Norgaard B, Kofoed PE, Kyvik KO, Ammentorp J. Communication skills training for health care professionals improves the adult orthopaedic patient's experience of quality of care. Scandinavian Journal of Caring Sciences. [Internet]. 2012 [citado em 2018 jun. 28]; 12: 698-704. doi: http://dx.doi. org/10.1111/j.1471-6712.2012.00982.x

20. Granheim M, Shaw JM, Mansah M. The use of interprofessional learning and simulation in undergraduate nursing programs to address interprofessional communication and collaboration: an integrative review of the literature. Nurse Educ Today [Internet]. 2018 [cited 2018 jun 28];62:118-27. doi: https://doi. org/10.1016/j.nedt.2017.12.021

21. Moen EK, Naden D. Intensive care patients' perceptions of how their dignityis maintained: a phenomenological study. Intensive Crit Care Nurs [Internet]. 2015 [cited 2018 jun 28];1(5):285-93. doi: https://doi.org/10.1016/j.iccn.2015.03.003

22. Boissy A, Windover AK, Bokar D, Karafa M, Neuendorf K, Rothberg MB. Communication skills training for physicians improves patient satisfaction. J Gen Intern Med [Internet]; 2016 [cited 2018 jun 28];31(7):755-61. doi: https://doi.org/10.1007/s11606-016-3597-2

23. Martins YC, Pessoni A. Comunicação e saúde na enfermagem: um estudo bibliométrico. RECIIS, Rev. Eletronica Comun Inf Inov Saude [Internet]. 2015 [citado em 2017 out. 27];9(1):1-13. doi: http:// dx.doi.org/10.29397/reciis.v9i1.445

24. Pfaff $K$, Baxter $P$, Jack $S$, Pleog, J. An integrative review of the factors influencing new graduate nurse engagement in interprofessional collaboration. J Adv Nurs [Internet]. 2014 [cited 2018 jun 28];70(10):420. doi: https://doi.org/10.1111/jan.12195

25. Chant S, Jenkinson TIM, Randle J, Russell G. Communication skills: some problems in nursing education and practice. ] Clin Nurs [Internet]. 2002 [cited 2018 jun. 28];11:12-21. doi: https://doi.org/10.1046/ j.1365-2702.2002.00553.x

26. Azevedo AL, Araújo ST, Vidal VLL. Como o estudante de enfermagem percebe a comunicação com o paciente em saúde mental. Acta Paul Enferm [Internet]. 2015 [citado em 25 out. 2017]; 28:125-31. doi: http://dx.doi.org/10.1590/1982-0194201500022.

27. Rosenzweig M, Hravnak M, Magdic K, Beach M, Clifton M, Arnold R. Patient Simulation Laboratory for students in an acute care nurse practitioner program. Am J Crit Care [Internet]. 2008 [cited 2017 out 25];17(4):364-72. Available from: http://ajcc.aacnjournals.org/content/17/4/364.full.pdf

28. Naughton C, Beardb C, Tzouvarab V, Pegramb A, Verityb R, Eleyb R, et al. A feasibility study of dementia communication training based on the VERA framework for pre-registration nurses: Part II impact on student experience. Nurse Education Today [Internet]. 2018 [cited 2017 out 25];63:87-93. doi: https:// doi.org/10.1016/j.nedt.2018.01.024

29. Kryachkov DA, Yastrebova EB, Kravtsova OA. Magic of Innovation: New Techniques and Technologies in Teaching Foreign Languages. Newcastle: Cambridge Scholars Publishing; 2015.

30. Beaird G, Nye C, Thacker LR. The Use of Video Recording and Standardized Patient Feedback to Improve Communication Performance in Undergraduate Nursing Students. Clinic Simul Nurs [Internet]. 2017 [cited 2017 out. 25];13:176-85. doi: https://doi.org/10.1016/j.ecns.2016.12.005

31. Brown MD. Taxonomy for the technology domain. J Chiropr Educ [Internet]. 2011 [cited 2018 jun. 28];25:57. Available from: https://www.ncbi.nlm.nih.gov/pmc/articles/PMC3113625/ 
32. Díaz JL, Leal C, García JA, Hernández E, Adánez MG, Sáez A. Self-learning methodology in simulated environments (MAESC): elements and characteristics. Clin Simul Nurs [Internet];2016 [cited 2018 jun 28];12(7):268-74. doi: https://doi.org/10.1016/j.ecns.2016.02.011

33. Jeffries PR. The NLN Jeffries Simulation Theory. Philadelphia: National League for Nursing; 2016.

34. Kable AK, Arthur C, Levett Jones T, Searl KR. Student evaluation of simulation in undergraduate nursing programs in Australia using quality indicators. Nurs Health Sci [Internet]. 2013 [cited 2017 out 25];15:235-43. doi: https://doi.org/10.1111/nhs.12025

35. Campbell S, Pagano MP, O'shea ER, Conery C, Caron C. Development of the Health Communication Assessment Tool: Enhancing Relationships, Empowerment, and Power-Sharing Skills. Clin Simul Nurs [Internet]. 2013 [cited 2017 out 25];9:543-50. doi: https://doi.org/10.1016/j.ecns.2013.04.016

36. Myers K, Chou CL. Collaborative and Bidirectional Feedback Between Students and Clinical Preceptors: Promoting Effective Communication Skills on Health Care Teams. J Midwifery Women's Health [Internet]. 2016 [cited 2018 jun 28];61:S22-7. doi: https://doi.org/10.1111/jmwh.12505

37. Lobiondo-Wood G, Haber J. Nursing Research. St. Louis: Mosby; 2013.

38. Ferrer M, Alonso J, Prieto L, Plaza V, Monsó E, Marrades R et al. Validity and reliability of the St George's Respiratory Questionnaire after adaptation to a different language and culture: the Spanish example. Eur Respir J. [Internet]. 1996 [cited 2017 out 25];9:1160-6. Available from: http://erj.ersjournals.com/ content/erj/9/6/1160.full.pdf

39. Balaminut T, Landim CAP, Becker TAC, Santos ECB, Olivatto GM, Zanetti ML, et al. Adaptação cultural e confiabilidade para o Brasil do Automated Telephone Disease Management: resultados preliminares. Acta Paul Enferm [Internet]. 2012 [citado em 2017 out. 25];25(5):795-801. Disponível em: http://www. redalyc.org/articulo.oa?id $=307026618012$

40. Freitas N, Caltran MP, Dantas RAS, Rossi LA. Translation and cultural adaptation of the Perceived Stigmatization Questionnaire for burn victims in Brazil. Rev Esc Enferm USP [Internet]; 2014 [cited 2017 out 25];48(1):25-33. doi: http://dx.doi.org/10.1590/S0080-623420140000100003

41. Strazzieri-Pulido KC, Santos VLCG, Carville K. Cultural adaptation, content validity and inter-rater reliability of the "STAR Skin Tear Classification System". Rev Latino-Am Enf [Internet]; 2015 [cited 2017 out 25];23(1):155-61. doi: http://dx.doi.org/10.1590/0104-1169.3523.2537

42. Epstein J, Santo RM, Guillemin F. A review of guidelines for cross-cultural adaptation of questionnaires could not bring out a consensus. J Clinic Epidemiol [Internet]. 2015 [cited 2017 out 25];68(4):435-41. doi: https://doi.org/10.1016/j.jclinepi.2014.11.021

43. Ferreira L, Neves AN, Campana MB, Tavares MCGCF.Guia da AAOS/IWH: sugestões para adaptação transcultural de escalas. Aval Psicol [Internet];2014 [citado 2017 out. 25]13(3):457-61.

44. Zandoná C, Cabral FB, Sulzbach CC. Produtivismo acadêmico, prazer e sofrimento: um estudo bibliográfico. Perspectiva [Internet]; 2014 [citado 25 out. 2017];38(144):121-30. Disponível em: http:// repositorio.ufsm.br/handle/1/12475

45. Rezende RC, Oliveira RMP, Araújo STC, Guimarães TCF, Santo FHE, Porto IS. Expressões corporais no cuidado: uma contribuição à Comunicação da Enfermagem. Rev Bras Enferm [Internet]; 2015 [citado 2017 out. 25]68(3):490-6. doi: http://dx.doi.org/10.1590/0034-7167.2015680316i 